\title{
Morfometría y faneroptica de subpoblaciones de cobayos (Cavia porcellus) nativos del altiplano sur ecuatoriano
}

\author{
Morphometric and faneroptic characterization of sub populations native cobayos \\ (Cavia porcellus) of the south equatorian highland
}

\author{
Cornelio Rosales-Jaramillo ${ }^{1^{\star}}$ iD, Rafael Róman-Bravo² iD y José Aranguren-Méndez²

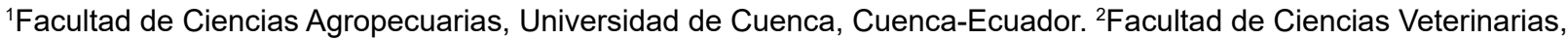 \\ Universidad del Zulia. Maracaibo-Venezuela. Correo Electrónico: cornelio.rosales@ucuenca.edu.ec
}

\section{RESUMEN}

El Cuy o Cobayo es un roedor nativo de los Andes sudamericanos de gran importancia para la identidad y seguridad alimentaria de la población, muy cotizado actualmente por las características únicas de su carne. La caracterización morfométrica y faneróptica de las especies animales nativas es el inicio de todo proceso racional de conservación de los recursos zoogenéticos y uso sostenible de las mismas para preservar su variabilidad genética y capacidad de resiliencia. Esta investigación se realizó en el altiplano sur de Ecuador. El objetivo consistió en realizar la caracterización morfométrica y faneróptica de dos subpoblaciones de cobayos (Cavia porcellus) nativos: Cañar (G1EcC), Azuay (G2EcA) y una línea mejorada Perú (G3LP); se registraron datos de 509 animales al nacimiento, 391 al destete y 370 a los 90 días. Para el análisis estadístico se aplicó estadística descriptiva, análisis de varianza con test de Duncan al $5 \%$ para datos con normalidad y Kruskal-Wallis y Mann-Whitney para datos sin normalidad. Las subpoblaciones nativas son animales corporalmente más pequeños comparados con los animales mejorados, con presencia de polidactilia, ojos de color negro, con una capa de tipo lacio corto y colores overo - agouti predominantes.

Palabras clave: Cobayo; zoogenéticos; fenotipo; características fanerópticas

\begin{abstract}
The guinea pig is a native rodent of the South American Andes of great importance for the identity and food security of the population, very much quoted at present for the unique characteristics of its meat. The morphometric and phaneroptic characterization of native animal species is the beginning of any rational process of conservation of animal genetic resources and their sustainable use to preserve their genetic variability and resilience. This research was carried out in the Southern Highlands of Ecuador. The objective was to perform the morphometric and phaneroptic characterization of two subpopulations of native guinea pigs (Cavia porcellus): Cañar (G1EcC), Azuay (G2EcA) and an improved line Peru (G3LP); data were recorded on 509 animals at birth, 391 at weaning and 370 at 90 days. For the statistical analysis, descriptive statistics were applied, analysis of variance with the Duncan test at $5 \%$ for normal data and Kruskal-Wallis and MannWhitney for data without normality. The native subpopulations were smaller animals as compared to the improved animals, with the presence of polydactyly, black eyes, with a short straight type coat and predominant overo - agouti colors.
\end{abstract}

Key words: Guinea pig; zoogenetics; phenotypes; phaneroptic characteristics 


\section{INTRODUCCION}

El Cobayo (Cavia porcellus) roedor histricomorfo de la familia Caviidae, es un mamífero originario de la zona andina sudamericana, especialmente de Bolivia, Colombia, Ecuador y Perú. Se le conoce también con los nombres de cuy, conejillo de Indias, cobayo, acure; es apreciado como alimento de alto valor nutricional que contribuye a la seguridad alimentaria de las familias rurales y urbanas aportando importantes nutrientes, lo que ha influido para que haya ganado mercado y aceptación global $[4,6]$.

El origen del Cobayo yace desde la antigüedad, citándose restos arqueológicos de cobayos silvestres, encontrados en Colombia, Perú y Chile y que datan entre los 9.000 a 8.000 años antes de Cristo (AC), siendo de estos hallazgos los más antiguos, los encontrados en la Sabana Bogotana de Tequendama en Colombia. Según algunos investigadores, el Cobayo constituye el roedor que más tempranamente fue domesticado, entre los 6.000 a 2.000 AC $[8,17,18]$.

En América del Sur, la domesticación de animales se concentra básicamente en cuatro especies, siendo una de ellas el Cobayo, evidencia ésta, dada por la abundancia de restos encontrados de esta especie, presumiendo su domesticación en esta región alrededor de los $2.500 \mathrm{AC}$, aunque existen evidencias, que se haya consumido su carne mucho antes, a través de la cacería de animales silvestres [17].

Estudios recientes basados en secuencias de mitogenoma proponen la existencia de dos núcleos principales de domesticación, muy probablemente originados con especies diferentes: el primero ubicado en Colombia basado en el Cavia anolaimae, de no muy lejano reconocimiento y otro núcleo, éste de origen andino central concentrado en el sur de Perú y norte de Chile, basado en el Cavia tschudii [18]; sin embargo, con el descubrimiento en la región central de Ecuador de la especie Cavia patzelti, no sería extraño que haya existido un tercer núcleo o proceso de domesticación [9].

Su clasificación taxonómica corresponde a:

Reino: Animalia

Subreino: Bilateria

Infrareino: Deuterostomia

Tipo: Chordata

Subtipo: Vertebrata

Infratipo: Gnathostomata

Superclase: Tetrapoda

Clase: Mammalia

Subclase: Theria

Infraclase: Eutheria

Orden: Rodentia

Suborden: Hystricomorpha

Infraorden: Hystricognathi

Familia: Caviidae

Subfamilia: Caviinae

Género: Cavia

Especie: Cavia porcellus

[15]
La población total real de Cobayos en Sudamérica, según censos agropecuarios entre 2013 y 2017 superan a los 100 millones (mill) de animales en Latinoamérica, distribuidos en Perú 65 mill [14], Ecuador 47 mill [13], Bolivia 3 mill [1] y Colombia 2,3 mill [10]; no obstante, la población en la actualidad se estiman serian de en Perú 17,38 mill, Ecuador 21,0 mill, Bolivia 0,647 mill y Colombia 1,234 mill de animales, de acuerdo a estimaciones de asociaciones no oficiales [20].

Los países ven reflejada su identidad cultural y tradiciones en la flora y fauna, por ello la variabilidad de los organismos en los ecosistemas, intraespecie o entre ellas, es un elemento importante para el desarrollo de la seguridad alimentaria y sostenibilidad de los pueblos. El menoscabo de esta variabilidad, acarrea la pérdida de dicha identidad, así como una parte del patrimonio de la humanidad y pone en riesgo la seguridad y autonomía alimentaria lo que obliga a propiciar el uso sostenible y la conservación de los recursos zoogenéticos para la agricultura y alimentación [21]. Un claro ejemplo lo constituye la crianza de los Cobayos por el hombre andino, destinándolo principalmente como medio de fuente de proteína, es decir para el consumo de su carne e incluso en algunas zonas su piel, es utilizada para hacer ropa, tal como se da en la sierra ecuatoriana $[2,6]$.

La conservación de los recursos zoogenéticos locales es una estrategia para garantizar la diversidad de los recursos buscando su uso sostenible para equilibrar las necesidades actuales y los objetivos de las generaciones futuras [7], además de propender a la preservación de los materiales biogenéticos de los animales domésticos que asegure su perpetuidad a lo largo del tiempo sin perder este valioso material [19].

A nivel mundial, los problemas reconocidos que enfrentan los recursos genéticos de animales criollos son los siguientes:

a) La disminución de la variabilidad genética dentro de razas o líneas de alta producción empleadas en sistemas intensivos de producción. b) La rápida desaparición de razas locales debido a la introducción de razas exóticas.

Frente a la problemática planteada, la Organización de las Naciones Unidas para la Alimentación y la Agricultura (FAO) [12] plantea desarrollar programas de acción para el manejo sostenible de la genética animal, su preservación (in situ y ex situ) y la formación de bancos de datos de recursos genéticos locales [11], considerando un protocolo lógico de acciones que permitan la conservación de poblaciones en peligro de extinción. Esta propuesta considera cinco fases iniciándose por la descripción general de la población en estudio, en donde se incluye precisamente la descripción de las características raciales, productivas, reproductivas, ecológicas, entre otras.

Para tal propósito se recomienda iniciar con la caracterización animal, siendo ésta la determinación de los atributos estructurales o funcionales de un individuo o grupo de individuos que particulariza a las líneas/razas o especies [12, 24]. Para ello, la morfología se determina de dos formas: atendiendo a los caracteres morfológicos o fanerópticos que son de naturaleza cualitativa (capa, color de ojos, de orejas, entre otras.) o atendiendo a los de estructura, que son cuantitativos y por lo tanto, factibles de medir a través de mediciones corporales concretas que cuantifican la conformación corporal [7, 24], información fundamental para procesos de selección y mejoramiento sostenibles en el propósito de formar 
líneas genéticas mejoradas, partiendo de germoplasma nativo buscando mantener la aptitud de resiliencia pero satisfaciendo las necesidades tanto económicas como alimenticias de productores y consumidores [4-6, 23].

En base a este lineamiento de la FAO, se planteó el objetivo principal de esta investigación, que consistió en describir las características morfométricas y fanerópticas de dos poblaciones de Cobayos ecuatorianos nativos (Cañar y Azuay) pertenecientes a subpoblaciones nacionales, para acercarse al conocimiento de su morfología y comportamiento en general y a su vez compararlos con una línea comercial mejorada (Perú).

\section{MATERIALES Y METODOS}

El estudio se llevó a cabo en la granja experimental de Irquis, de la Facultad de Ciencias Agropecuarias de la Universidad de Cuenca, ubicada en el altiplano sur del Ecuador, cuyas coordenadas son: $4^{\circ} 48^{\prime} 34^{\prime \prime}$ LS y $79^{\circ} 4^{\prime} 30.12^{\prime \prime}$ LO; a una altitud de 2.664 metros sobre el nivel del mar (m.s.n.m.), humedad relativa del $80 \%$, pluviosidad entre 800 - 2000 milímetros (mm) y temperatura que oscila entre 7 y $12^{\circ} \mathrm{C}$.

Los datos se registraron en diferentes etapas de la vida de los animales. Se usaron al nacimiento, 211 descendientes de la subpoblación Cañar (G1EcC) (FIG.1A), 143 descendientes de la subpoblación Azuay (G2EcA) (FIG.1B) y 155 descendientes de línea Perú (G3LP); al destete 166 (G1EcC), 113 (G2EcA) y 112 (G3LP); y a los 90 días (d) 157 (G1EcC), 108 (G2EcA) y 105(G3LP), todos ellos mantenidos bajo un mismo sistema de crianza, de manejo y alimentación. Las medidas fueron tomadas en base a la metodología establecida por Tirira [26] y con el uso de un calibrador digital Vernier SHARS Aventor® 8»/200mm, EUA) y una balanza de precisión BPS 52 plus $B O E C O \circledast$, Alemania) con precisión de 0,01 gramo (g).

Las variables morfométricas y fanerópticas determinadas fueron: largo total del cuerpo (LT), medida que va desde el extremo del hocico hasta el extremo de la cola; largo de la cabeza (LC) desde el extremo del hocico hasta la nuca; largo de la pata posterior (LP), desde el talón hasta la punta de la garra; largo de la oreja (LO), desde su base hasta la punta, por la cara anterior; alzada de cabeza $(A C)$; peso individual $(P)$; número de dedos en los miembros anteriores (NDA); número de dedos en los miembros posteriores (NDP); tipo de pelaje (TP) si eran lisas, enrosetadas o ensortijadas (FIG. 2); color de ojos (CO); color de orejas (COr); color de capa (CC) las cuales se clasificaron en: simple que implica un solo color entero y firme, el overo dos colores firmes claramente definidos y el compuesto o agouti formado por combinaciones indefinidas de pelos de diferente color (FIGS. 3 y 4 ).

El análisis estadístico se realizó aplicando estadística descriptiva, tablas de frecuencia, histogramas, además prueba de ShapiroWilk para normalidad, ANAVA a una vía de clasificación, para comparar las poblaciones se utilizaron prueba de Duncan $(P<0,05)$ y para aquellas variables cuyos residuos no se ajustaron a una distribución normal se usaron las pruebas de KruskalWallis y Mann-Whitney, todo ello utilizando el paquete estadístico SAS 9.0 [25].

\section{RESULTADOS Y DISCUSION}

De acuerdo a los datos encontrados (TABLA I) se observó que las subpoblaciones Azuay (G2EcA) y Cañar (G1EcC) responden a una morfología externa diferente en todos los parámetros estudiados, a excepción del largo de oreja en donde no muestran diferencias estadísticas $(P>0,05)$. Se encontraron diferencias significativas con valores a favor de la subpoblación Cañar, mostrándose como un animal de mayor tamaño y peso en todas las etapas de la vida estudiadas pasando de un largo de cuerpo de 12,42 centímetros $(\mathrm{cm}) \pm 0,09$ y un peso de $113,52 \mathrm{~g} \pm 2,51$ al nacimiento a 23,63 cm $\pm 0,18$ y $667,88 \mathrm{~g} \pm 10,45$ a los $90 \mathrm{~d}$ de edad frente a $11.91 \mathrm{~cm} \pm 0,08$ y un peso de $102,62 \mathrm{~g} \pm 2,23$ al nacimiento, a 21,12 cm $\pm 0,23$ y 512,6 g $\pm 13,41$ a los $90 \mathrm{~d}$ de edad en la variedad Azuay.
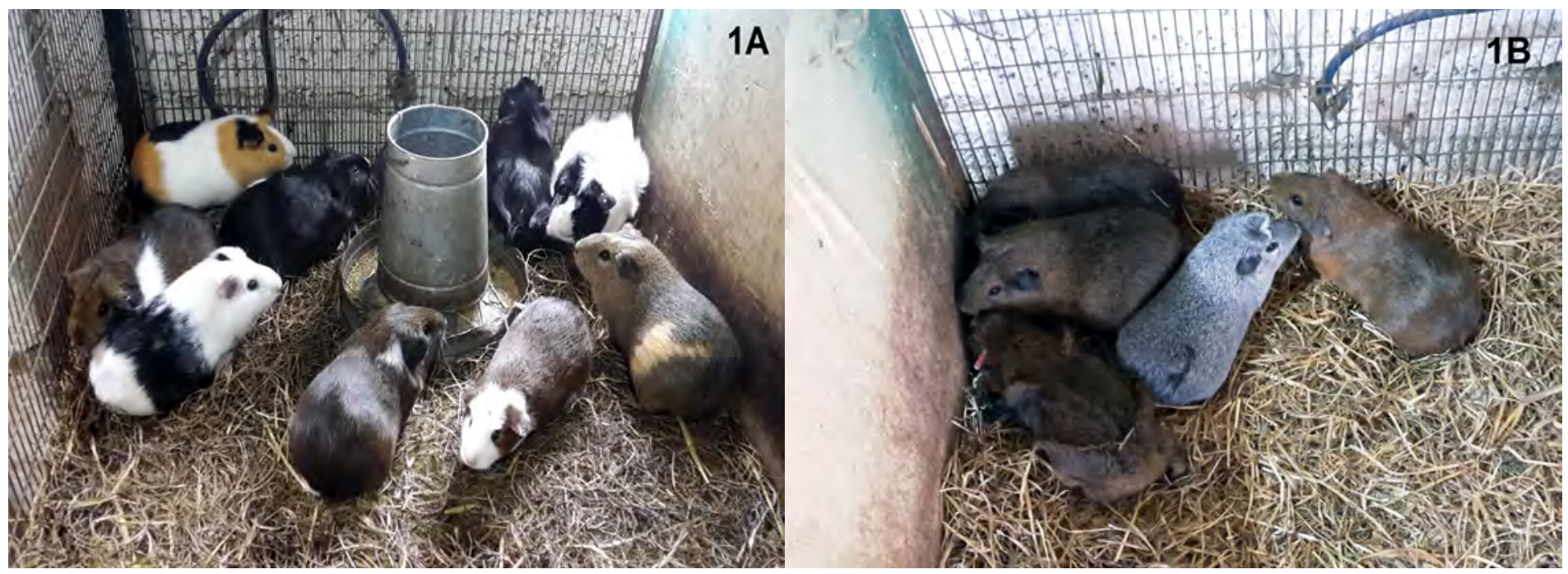

FIGURA 1. Subpoblación Cañar (1A) y subpoblación Azuay (1B) de cobayos nativos del altiplano sur del Ecuador 
Morfometria y faneroptica de cobayos nativos / Rosales-Jaramillo y col.

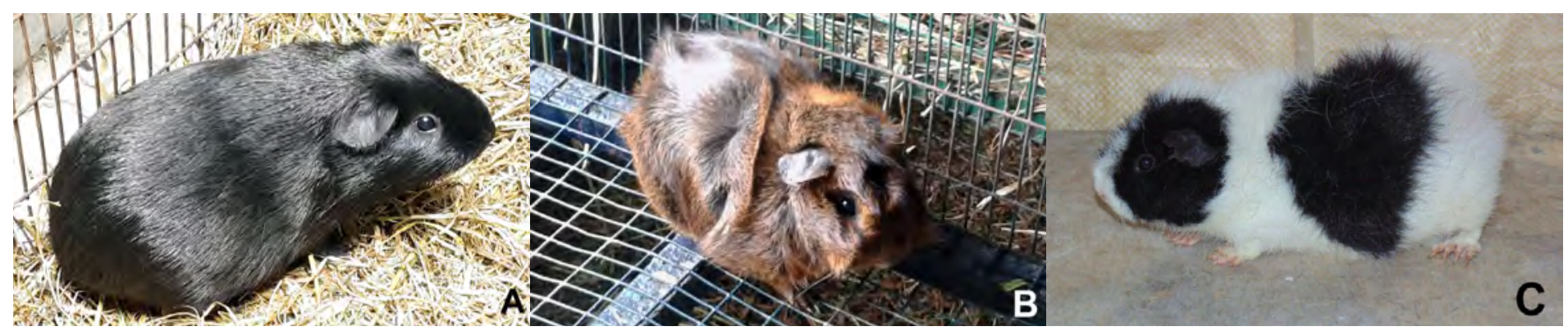

FIGURA 2. Ejemplar de cobayo nativo del altiplano sur del Ecuador, con pelo liso (A), pelo enrosetado (B) y ensortijado (C)
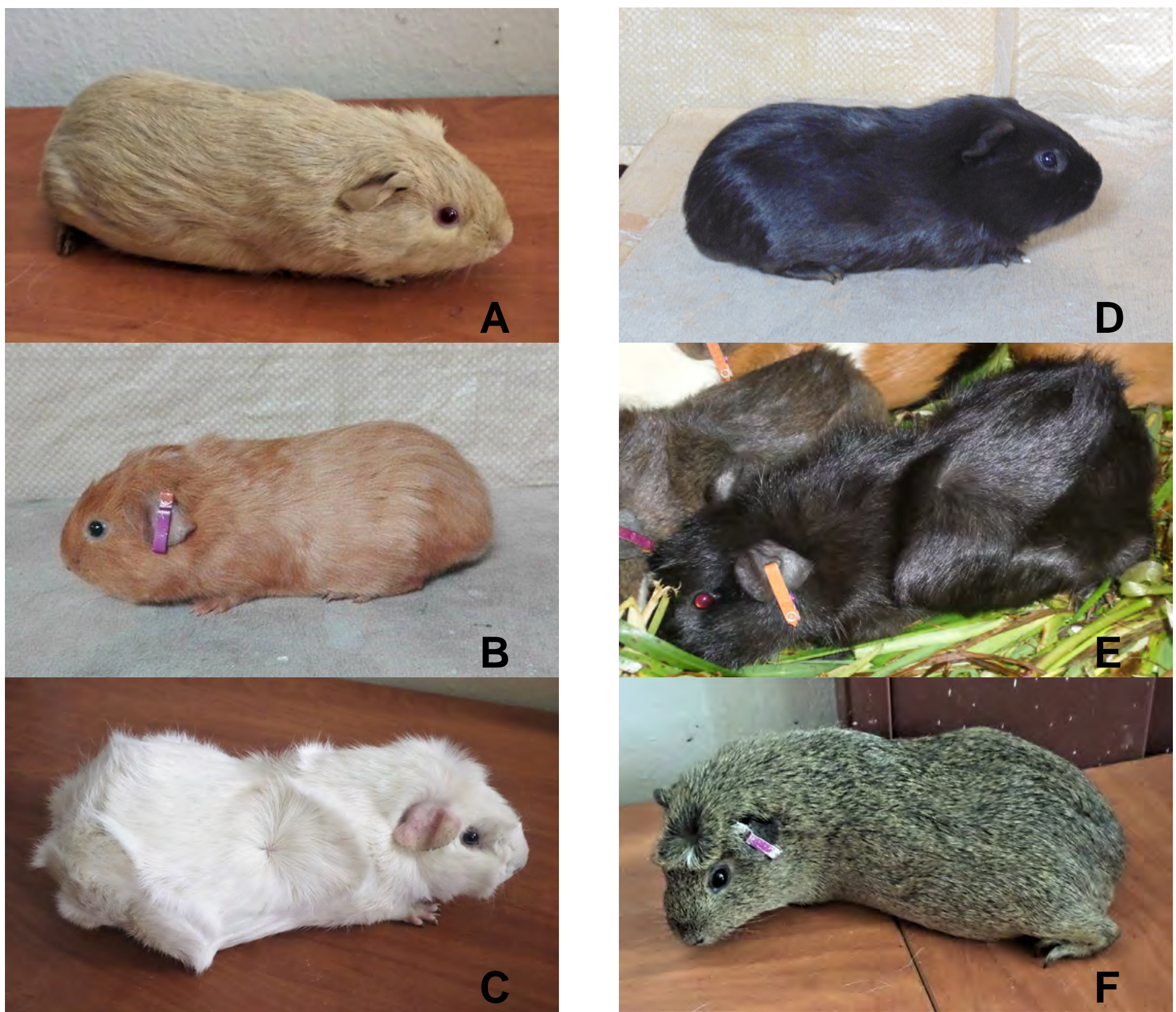

FIGURA 3. Principales colores de capa solida: Bayo (A), Alazán (B), Blanco (C), Violeta (D), Negro (E) y Agoutí crema (F), presentados en subpoblaciones de cobayos nativos del altiplano sur del Ecuador 

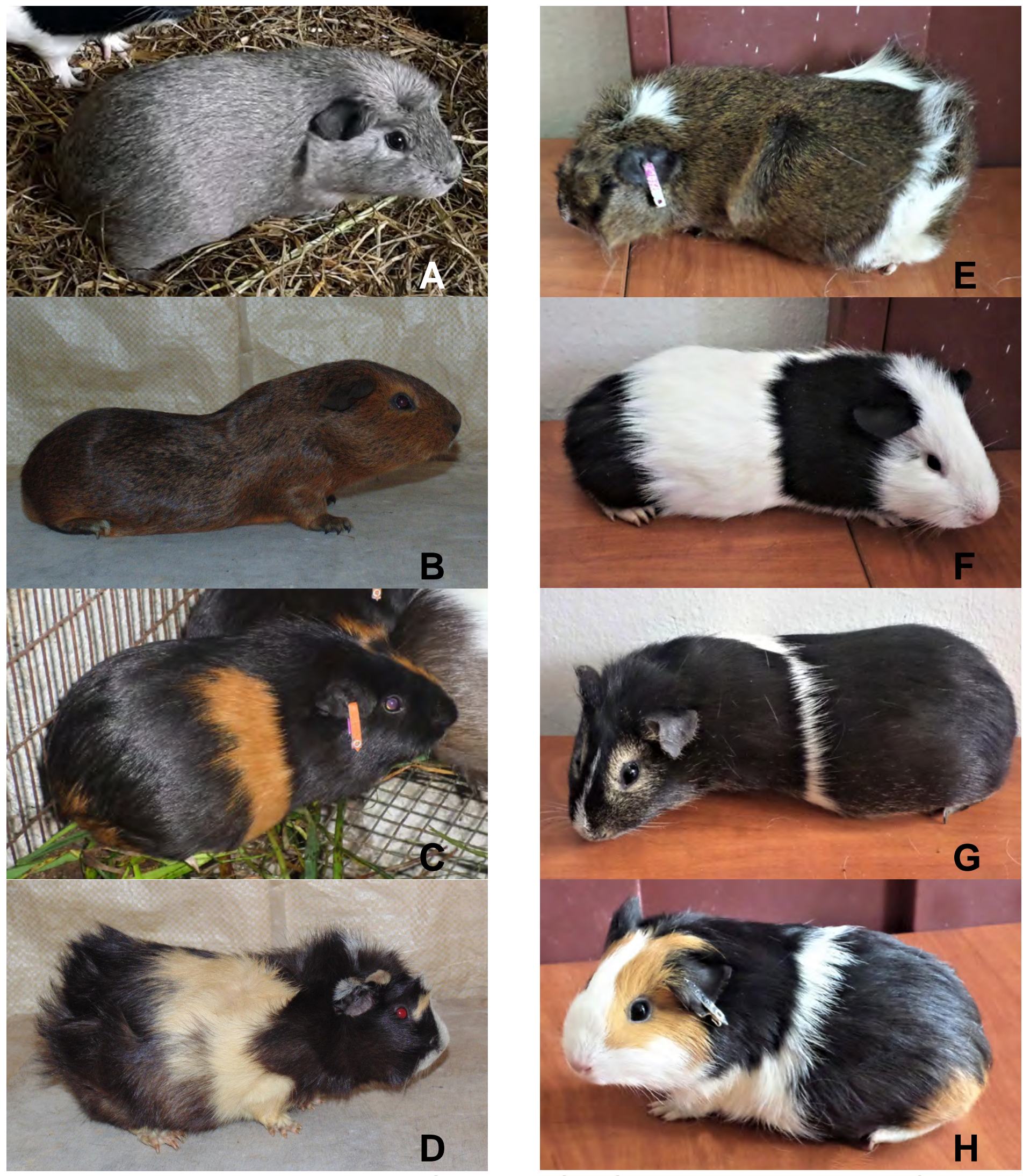

FIGURA 4. Principales colores de capa solida: Agoutí gris (A), Agoutí marrón (B) y capa combinada: Negro alazán (C), Negro bayo (D), Agoutí overo (E), Overo negro (F), Negro overo (G) y Combinado (H), presentados en subpoblaciones de cobayos nativos del altiplano sur del Ecuador 
Morfometria y faneroptica de cobayos nativos / Rosales-Jaramillo y col.

TABLA I

Indicadores morfométricos y fanerópticos de cobayos nativos Azuay, Cañar y línea Perú

\begin{tabular}{|c|c|c|c|c|}
\hline Variable & Procedencia & $\begin{array}{c}\text { Al nacimiento } \\
\overline{\mathrm{X}} \pm \mathrm{EE}\end{array}$ & $\begin{array}{c}\text { Al destete } \\
\bar{X} \pm E E\end{array}$ & $\begin{array}{c}\text { A los } 90 \text { días } \\
\bar{X} \pm E E\end{array}$ \\
\hline \multirow{3}{*}{$\begin{array}{l}\text { Largo total } \\
\text { cm. }\end{array}$} & G1EcC & $12,42 \pm 0,09^{b}$ & $16,11 \pm 0,18^{b}$ & $23,63 \pm 0,18^{b}$ \\
\hline & G2EcA & $11,91 \pm 0,08^{c}$ & $15,13 \pm 0,17 c$ & $21,12 \pm 0,23 c$ \\
\hline & G3LP & $13,49 \pm 0,15^{a}$ & $17,14 \pm 0,29 a$ & $26,42 \pm 0,22^{a}$ \\
\hline \multirow{3}{*}{$\begin{array}{l}\text { Largo de cabeza } \\
\text { cm. }\end{array}$} & G1EcC & $4,79 \pm 0,03 b$ & $5,28 \pm 0,04^{b}$ & $6,77 \pm 0,03^{b}$ \\
\hline & G2EcA & $4,56 \pm 0,03^{c}$ & $5,18 \pm 0,04^{b}$ & $6,39 \pm 0,04^{c}$ \\
\hline & G3LP & $5,20 \pm 0,05^{a}$ & $5,82 \pm 0,07 a$ & $7,36 \pm 0,04^{a}$ \\
\hline \multirow{3}{*}{$\begin{array}{l}\text { Alzada de cabeza } \\
\text { cm. }\end{array}$} & G1EcC & $4,53 \pm 0,04^{b}$ & $5,01 \pm 0,04^{b}$ & $6,46 \pm 0,04^{b}$ \\
\hline & G2EcA & $4,31 \pm 0,03^{c}$ & $4,82 \pm 0,04^{c}$ & $6,02 \pm 0,05^{c}$ \\
\hline & G3LP & $4,84 \pm 0,06^{a}$ & $5,49 \pm 0,06^{a}$ & $6,93 \pm 0,05^{a}$ \\
\hline \multirow{3}{*}{$\begin{array}{l}\text { Largo de oreja } \\
\text { cm. }\end{array}$} & G1EcC & $2,27 \pm 0,03^{b}$ & $2,51 \pm 0,04^{b}$ & $3,13 \pm 0,11 a b$ \\
\hline & G2EcA & $2,21 \pm 0,03^{b}$ & $2,50 \pm 0,04^{b}$ & $2,91 \pm 0,14^{b}$ \\
\hline & G3LP & $2,57 \pm 0,05^{a}$ & $2,73 \pm 0,07^{a}$ & $3,34 \pm 0,14^{a}$ \\
\hline \multirow{3}{*}{$\begin{array}{l}\text { Largo de pata } \\
\text { cm. }\end{array}$} & G1EcC & $3,63 \pm 0,03 b$ & $3,83 \pm 0,02 \mathrm{~b}$ & $4,53 \pm 0,02 b$ \\
\hline & G2EcA & $3,47 \pm 0,02^{c}$ & $3,70 \pm 0,02^{c}$ & $4,26 \pm 0,03 c$ \\
\hline & G3LP & $4,18 \pm 0,04^{a}$ & $4,27 \pm 0,04^{a}$ & $5,13 \pm 0,03 a$ \\
\hline \multirow{3}{*}{$\begin{array}{l}\text { Peso } \\
\text { g. }\end{array}$} & G1EcC & $113,52 \pm 2,51 b$ & $224,15 \pm 5,48 b$ & $667,88 \pm 10,45 b$ \\
\hline & G2EcA & $102,62 \pm 2,23^{c}$ & $203,08 \pm 5,32^{c}$ & $512,66 \pm 13,41 c$ \\
\hline & G3LP & $150,26 \pm 4,08^{a}$ & $289,28 \pm 8,71^{a}$ & $1011,16 \pm 12,92^{a}$ \\
\hline \multirow{3}{*}{$\begin{array}{c}\text { Número de dedos } \\
\text { anteriores }\end{array}$} & G1EcC & $4,34 \pm 0,07^{c}$ & - & - \\
\hline & G2EcA & $4,17 \pm 0,06^{b}$ & - & - \\
\hline & G3LP & $3,98 \pm 0,11 \mathrm{a}$ & - & - \\
\hline \multirow{3}{*}{$\begin{array}{l}\text { Número de dedos } \\
\text { posteriores }\end{array}$} & G1EcC & $3,69 \pm 0,08^{b}$ & - & - \\
\hline & G2EcA & $3,53 \pm 0,08^{b}$ & - & - \\
\hline & G3LP & $3,05 \pm 0,02 \mathrm{a}$ & - & - \\
\hline
\end{tabular}

Letras diferentes en columna indican diferencia significativa $(P<0,05)$

Estos valores encontrados comparados con los descritos por Kunz [16], para Cobayos amerindios resultan ser inferiores, por el contrario, otro estudio en variables similares en Cobayos negros encontró valores inferiores a los descritos en la presente investigación [2]. No obstante, al comparar estas subpoblaciones locales (Cañar y Azuay) con animales de línea mejorada (Perú) se encuentra que son animales inferiores en toda su morfología a excepción del largo de oreja, con seguridad está determinado por los procesos de selección a los cuales ha sido sometida la especie, observándose que desde el nacimiento son animales de mayor tamaño y peso con valores de 1,07 y 1,58 cm y 36,74 y 47,64 g. más que Cañar y Azuay, respectivamente; dichas diferencias se hacen más marcadas a los $90 \mathrm{~d}$ en donde los animales ya han expresado todo su potencial productivo siendo 2,79 y $5,3 \mathrm{~cm}$ y 343,28 y 498,5 g más que Cañar y Azuay, respectivamente.

La polidactilia, factor considerado como causal de descarte en las líneas mejoradas [3, 27], aún se encuentra presente con relativa alta frecuencia en los animales nativos, tanto en extremidades anteriores como posteriores, en virtud de que en estos últimos, es muy poco el mejoramiento genético realizado y poco el control de los apareamientos, siendo muy común aquellos de orden consanguíneos $[19,20]$. El 23,2 \% de animales de origen Cañar presentaron polidactilia en los miembros anteriores frente a un $12,6 \%$ en Azuay y un 0,6 \% en la línea Perú; por el contrario, Azuay presentó un mayor porcentaje 33,6 \% en los miembros posteriores frente a un 29,9 y 4,5\% en Cañar y mejorado, respectivamente, estos valores difieren de los mencionados por 
otro autor [22], observándose que los nativos bolivianos presentan mayor frecuencia de polidactilia $30 \%$ en los miembros anteriores que los nativos ecuatorianos, por el contrario presentan menor frecuencia $(26,6 \%)$ en los miembros posteriores, de igual forma otros investigadores encontraron un porcentaje promedio mayor (45\%) de polidactilia en cobayos del cantón Loja [3].

En cuanto al número de dedos, los animales nativos muestran un mayor promedio, la subpoblación Cañar $(3,69 \pm 0,08)$ sobresale con un 16,6 y $14,7 \%$ de animales con 6 dedos en extremidades anteriores y/o posteriores, respectivamente; en Azuay $(3,53 \pm 0,08)$ sobresale un $8,4 \%$ para miembros anteriores con 6 dedos y un $21,2 \%$ con 4 dedos en miembros posteriores, encontrándose un máximo de 7 dedos anteriores y posteriores en un porcentaje menor al $1 \%$ únicamente en cobayos nativos. Los hallazgos son similares a los mencionados por Rico [23], en Cobayos nativos bolivianos, especialmente en los miembros anteriores Cobayo promedio es 4,33 ya que en los posteriores presentan un menor promedio de 3,28 al encontrado en los nativos ecuatorianos.

En cuanto al tipo de pelo (FIG. 5), el corto y lacio es el predominante en las subpoblaciones estudiadas, encontrándose el 83,4 y $76,2 \%$ en los animales de procedencia Cañar y Azuay, respectivamente; mientras que, los porcentajes restantes corresponden a animales con pelo corto y arrosetado, estos tipos de pelo se corresponden con los encontrados en otras investigaciones [23]; la línea Perú solo presenta pelo corto y liso.

Las subpoblaciones nativas Cañar y Azuay se caracterizan por presentar una capa de color overo y agoutí encontrándose este último exclusivamente en los Cobayos nativos (FIG. 6). En Cañar, el $8,1 \%$ de animales presentaron una capa de color simple con presencia de negro, blanco y alazán, el $47,4 \%$ fueron overos con presencia de blanco en combinación con el agoutí, negro, alazán y viceversa, por último, un $44,5 \%$ presentó una capa compuesta

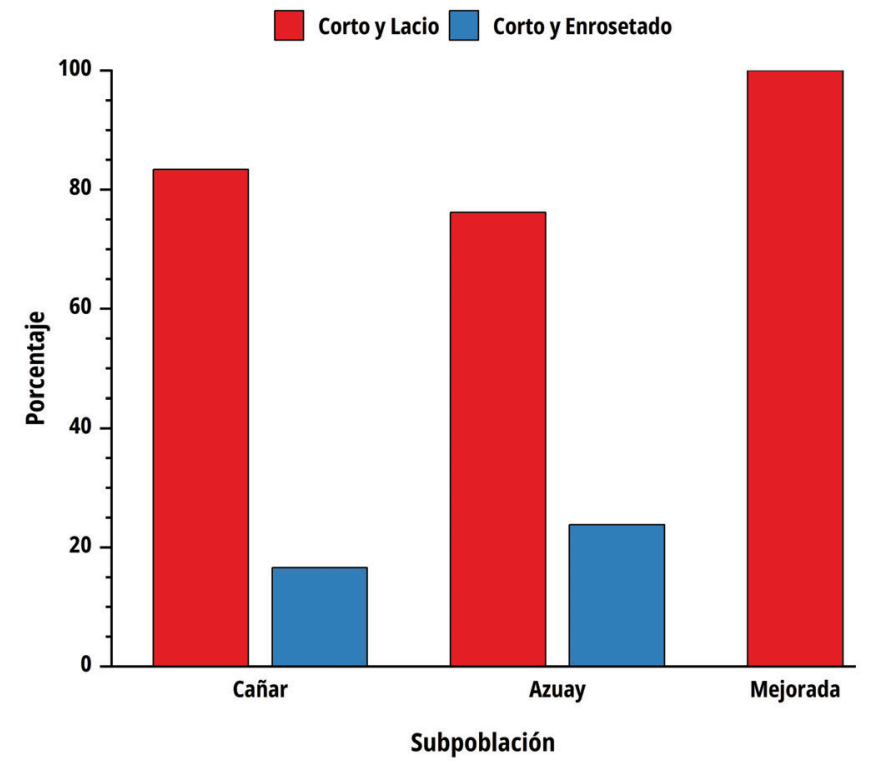

FIGURA 5. Composicion de subpoblaciones de acuerdo al tipo de pelo

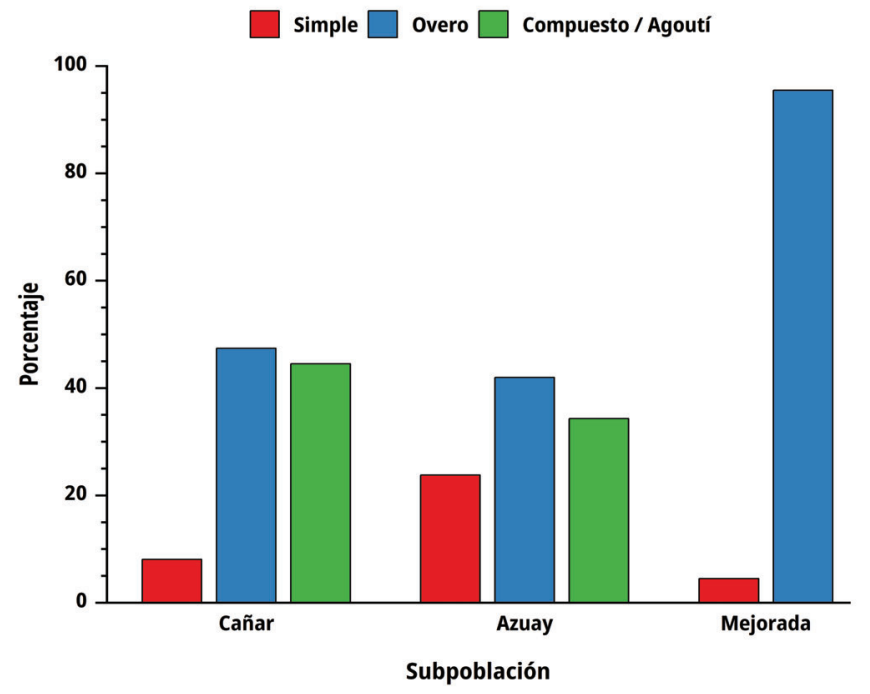

FIGURA 6. Composicion de subpoblaciones de acuerdo al color de la capa

o agoutí siendo ruano, lobo, moro y más de 3 colores definidos blanco, bayo, alazán, negro, plomo, agoutí. En Azuay, el 23,8 \% de animales presentaron una capa simple, el mayor porcentaje $42 \%$ fueron overos en ambos grupos con colores iguales a Cañar y para el color de capa compuesta el porcentaje fue de $34,3 \%$ con combinaciones de colores marrón, lobo, ruano, moro, todos los colores hallados son coincidentes con los descritos anteriormente [23]; la línea Perú debido a los procesos de selección el color overo es el predominante en donde el más representativo fue el alazán con blanco y el restante fue para colores simples como el blanco, bayo y alazán.

En cuanto al color de ojos, en las tres subpoblaciones prevalece el color negro existiendo apenas un $2 \%$ de color rojo, valor similar al mencionado previamente [22]. El color de oreja negra es el más común en las tres subpoblaciones, tanto en la oreja izquierda como en la derecha, seguido del moteado y el menor porcentaje de animales presenta oreja rosada (FIG. 7); no siempre existe coincidencia de color de oreja izquierda y derecha en los animales.

\section{CONCLUSIONES}

A partir de la información analizada, se puede indicar que las subpoblaciones nativas estudiadas presentan características diferentes entre sí, son de menor peso y tamaño corporal en comparación con la línea mejorada Perú, con presencia de polidactilia en las patas delanteras y traseras debido a los procesos de selección poco rigurosa y posible consanguinidad presentes en los sistemas tradicionales de crianza en ellos prevalece el pelo lacio y corto, con color de capa diversa con alta presencia de overos y agoutí color típico de los animales silvestres, acompañado con ojos y orejas de color negro; todas las características presentes hacen prever todavía la existencia de una considerable variabilidad genética. 


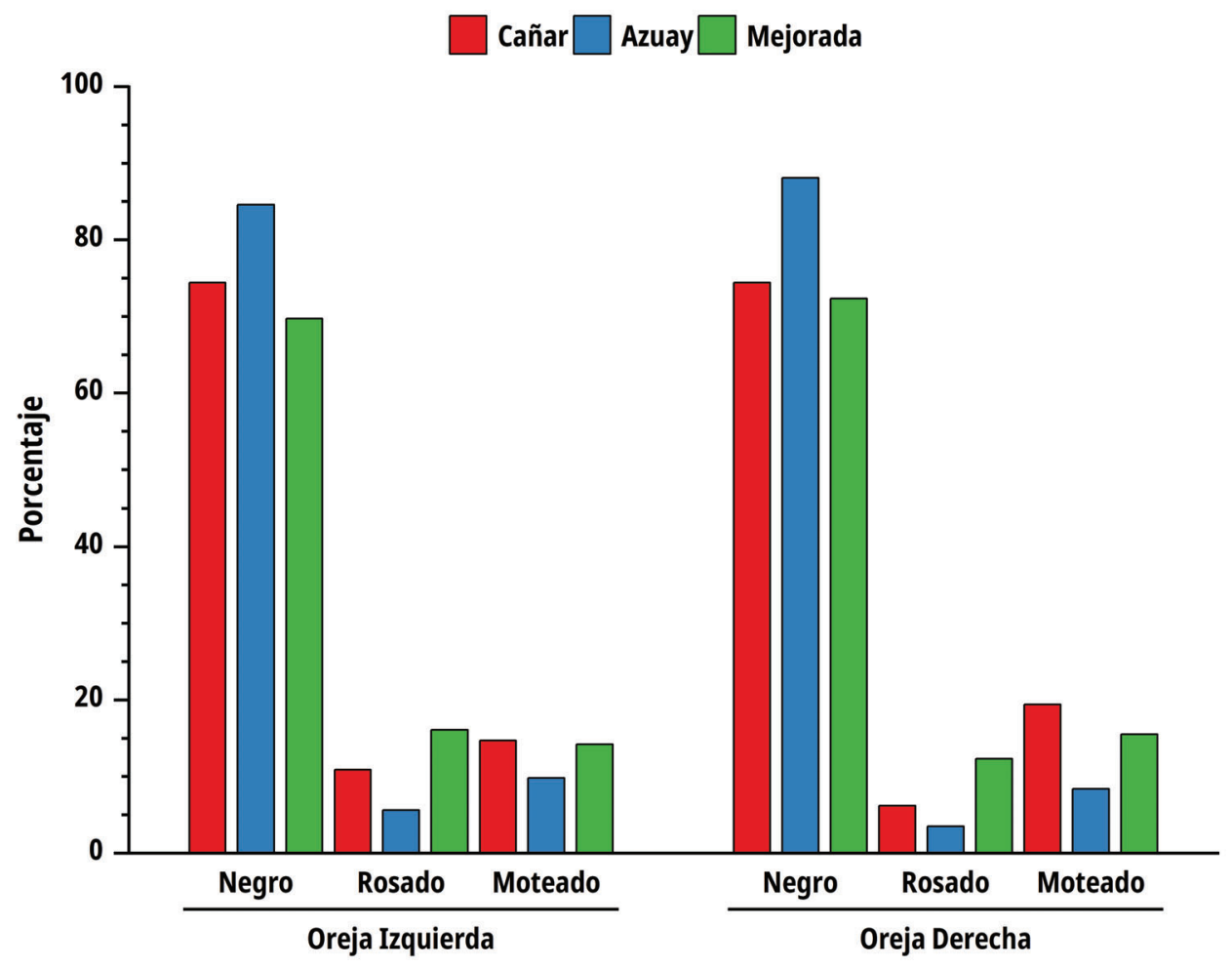

FIGURA 7. Composicion de subpoblaciones de acuerdo al color de las orejas

\section{AGRADECIMIENTO}

Especial mención a la Dirección de Investigación de la Universidad de Cuenca (DIUC), Cuenca-Ecuador, por el financiamiento de esta investigación.

\section{REFERENCIAS BIBLIOGRAFICAS}

[1] ARCHIVOS NACIONAL DE DATOS (ANDA). Censo Agropecuario del Estado Plurinacional de Bolivia, 2013. La Paz, Bolivia. En Línea: https://bit.ly/3gTtA82. 18/08/2020.

[2] BARRERA, A.; USCA, J.; DIAZ, H.; CASTILLO, B. Evaluación de las Características Productivas y Reproductivas de Cobayos Negros manejados en jaulas versus pozas. Rev. Carib. Cien. Social. 12 (3):103-107. 2018.

[3] BENITEZ, E.; ULLAGUARI, V.; GUZMAN, J.; ABAD, R. Estado de la polidactilia en poblaciones de cobayos (Cavia porcellus) en el cantón Loja, como indicador de su estatus genético. Centro Biotecnol. 6:36-40. 2017.

[4] CHAUCA, L. Producción de Cobayos (Cavia porcellus). En: Estudio FAO Produccion y Sanidad Animal No. 138 (Ed.) Roma - Italia. 134 pp. 1997.

[5] CHAUCA, L. Logros obtenidos en la mejora genética del Cobayo (Cavia porcellus). Experiencias del INIA. Arch. Latinoam. Prod. Anim. 15:218 - 222. 2007

[6] CHAUCA, L. Crianza del Cobayo Cavia porcellus y su impacto en el desarrollo rural. Proc. XXXVIII Reunion de la
Asociacion Peruana de Produccion Anim. (APPA). Lima, 12/04-06, Peru. Pp: 64-69. 2013.

[7] CONSORCIO GTZ/FUNDECO/IE. Estrategia regional de biodiversidad. Protección, recuperación y difusión de conocimientos y prácticas tradicionales. (Eds.). BID \& Comunidad Andina de Naciones, Lima. 97 pp. 2001.

[8] DIGARD, J. Un Aspect méconnu de l'histoire de l'Amérique : la domestication des animaux. L'Homme. 32:253-270. 1992.

[9] DUNNUNM, J.; SALAZAR, J. Molecular systematics, taxonomy and biogeography of the genus Cavia (Rodentia: Caviidae). J. Zool. System. Evolut. Res. 48 (4): 376-388. 2010.

[10] ENCUESTA NACIONAL AGROPECUARIA (ENA). Oferta Agropecuaria, ENA - Cifras 2013. Bogotá, Colombia. En Línea: http://www.agronet. gov.co. 05/10/2020.

[11] FOOD AND AGRICULTURE ORGANIZATION (FAO). Lista Mundial de Vigilancia para la Diversidad de los Animales Domésticos. SCHERF, B. (Ed.) 2nd. Ed. FAO, Roma. 777 pp. 1997.

[12] FOOD AND AGRICULTURE ORGANIZATION (FAO). La situación de los recursos zoogenéticos mundiales para la alimentación y la Agricultura. PILLING, B.R. (Ed.) FAO, Roma. 555 pp. 2010.

[13] INSTITUTO NACIONAL DE ESTADISTICAS Y CENSO (INEC). III Censo Agropecuario. Ecuador. En Línea: http://www. inec.gov.ec. 18/10/2020. 
[14] INSTITUTO NACIONAL DE ESTADISTICA E INFORMATICA (INEI). Censo Agropecuario-CENAGRO 2017. Lima, Perú. En Línea: http://www.inei.gob.pe. 18/10/2020.

[15] INTEGRATED TAXONOMIC INFORMATION SYSTEM. ITIS Report. Base de datos Electronica de los Recursos On Line Animales. https://www.itis.gov/ 18-05-2020.

[16] KUNZ, M. Estudio Comparado de Variables Corporales y Tamaño de Camada en Cobayos (Cavia porcellus) Amerindios (Arica) y de Laboratorio desde el Nacimiento hasta los cuatro meses de edad. Facultad de Ciencias Veterinarias y Pecuarias - Universidad de Chile. Tesis de Grado. 345 pp. 2003.

[17] LAVALEÉ, D. La Domestication Animales En Amerique Du Sud - Le point des connaissances. Bull. Inst. Fr. Études Andines. 19 (1):25-44. 1990.

[18] LORD, E.; COLLINS, C.; DE FRANCE, S.; LEFEBVRE, M.; PIGIÉRE, F.; EECKHOUT, P.; ERAUW, C.; FITZPATRICK, S.M.; HEALY, P.; MARTINEZ-POLANCO, M.; GARCIA, J.L.; RAMOS, E.; DELGADO, M.; SANCHEZ, A.; PEÑA, G.; TOYNE, J.; DAHLSTED, A.; MOORE, K.; LAGUER, C.; ZORI, C.; MATISOOSMITH, E. Ancient DNA of Guinea Pigs (Cavia spp.) Indicates a Probable New Center of Domestication and Pathways of Global Distribution. Scientif. Rep. 10:8901-8908. 2020.

[19] MANTILLA, J. Mejoramiento Genético y Conservación de Cobayos Nativos en el Perú. Sistema de Revisiones en Investigación Veterinaria de San Marcos. Cajamarca, 08/1214, Peru. Pp 1-5. 2009.
[20] PATIÑO, R.; CARDONA, J.; CARLOSAMA, L.; PORTILLA, P.; MORENO, D. Zootechnical parameters of Cavia porcellus in production systems in Nariño and Putumayo (Colombia). Rev. CES Med. Zoot. 14 (3):29-41. 2019.

[21] RAYMONDI, J. Evaluación Genética de un Plantel de Cobayos Reproductores de Genotipo Perú. Rev. Inv. Vet. Perú. 28 (2): 293-298. 2017.

[22] RICO, E. Planteles de Cobayos locales e introducidos en Bolivia. Proc. Seminario Internacional: Avances en Investigación, Organización Empresarial, Producción, Procesamiento y Comercialización de Cobayos. Lima, 08/22-23, Peru. 11pp. 2010.

[23] RICO, E.; RIVAS, C. Conservación de poblaciones nativas de Cobayos. Rev. Invest. Agrop. Fores. Boliv. 1(2):49-56. 2017.

[24] SAÑUDO, C. Importancia de la Morfología y su valoración en los animales Domésticos. Valoración Morfológica de los Animales Domésticos. Ministerio de Medio Ambiente y Medio Rural y Marino Secretaría General Técnica Centro de Publicaciones (Ed.) Madrid. 863 pp. 2009

[25] STATISTICAL ANALYSIS SYSTEM INSTITUTE (SAS). Versión 9.0. User's guide: Statistics. Cary, NC. 2002.

[26] TIRIRA, D. Orden Rodentia: Roedores. Mamíferos del Ecuador: Guía de campo. Quito, Pichincha, Ecuador: Murciélago Blanco (Ed.) Pichincha. 576 pp. 2007.

[27] WRIGHT, S. An analysis of variability in number of digits in an inbred strain of guinea pigs. Genetics. 19: 506-536. 1934. 